\title{
Screening donors of solid organs for West Nile virus: First, do no harm!
}

\author{
Kevin Forward MD
}

$\mathrm{H}$ ealth Canada has recently published guidelines that promote testing donors of tissues and perfusable organs for West Nile virus (WNV) using nucleic acid amplification testing (NAT) (such as polymerase chain reaction) (1). It is my belief that the rationale underlying these guidelines is fundamentally flawed and that these guidelines will harm (or already have harmed) patients.

My primary concerns relate to polymerase chain reaction testing of donors of perfusable organs. There is no doubt that transplanted organs can transmit WNV and that infected recipients may experience particularly severe outcomes (2). The Health Canada guidelines suggest that all donors should have NAT testing performed before transplantation. These guidelines do not suggest that such testing be considered in the context of disease activity (either local or seasonal epidemiology). For instance, under these guidelines, a donor who has not left Manitoba for months should be tested for WNV at midnight on New Year's Eve.

The failure to consider the pretest probability of active infection, when combined with a lack of knowledge of the performance characteristics of available tests (which are licensed and have not been formally evaluated for this indication), is a recipe for disaster (eg, discarding life-saving organs).

\section{THE PRETEST PROBABILITY}

The chance that an organ donor might be viremic at the time of donation is remote, even in an outbreak setting. In Canada during the summer of 2003, the Canadian Blood Services identified only 14 blood donors who were viremic at donation, representing one in 8622 donors. In Saskatchewan, where WNV was known to be hyperendemic at the time, viremia was uncommon. Between July 21 and September 7, 2003, 10 of 7482 Saskatchewan donors tested were positive for WNV RNA. Last summer, no positive donors were identified in any of the Atlantic Provinces. Testing outside of WNV season and in areas where WNV is relatively inactive or nonexistent dramatically decreases the prevalence and, therefore, the pretest probability beyond the $1 / 8600$ average during the summer of 2003.

In 2004, there have been only 17 cases of WNV infections documented in Canada. This is unexpected, although Health Canada was aware of this fact by the time the guidelines were issued. The dramatic decrease in the incidence further challenges justification for these guidelines.

\section{THE PROBLEM WITH NAT TESTING}

Organ transplantation programs do not have access to the methodologies used to screen blood donors. Testing, therefore, is performed using unlicensed methodology not approved for the purpose of screening. Often, testing must be done on a 'stat' or callback basis. At this time, we simply do not know the performance characteristics of available NAT tests, at least to the degree of precision necessary for such an application. If one in 800 NAT tests are falsely positive and the true likelihood of viremia is one in 8000 , then only $10 \%$ of results would be true positives, and perhaps 40 organs might be discarded for every true positive test. Given the mortality rate on our transplantation waiting lists, it is probable that many patients would die as a result of these false positives (3).

When NAT testing is performed on a 'stat' basis with the transplantation team standing by, there would be little or no opportunity to confirm the results of the original test. Few laboratories have an alternate technology to confirm the initial result, and tests failing to confirm the original positive may still lead to organ refusal.

The logistics of NAT testing for organ donation present a number of problems. Because testing of organ donors needs to take place on a 'stat' basis, laboratories will be obliged to have specially trained technologists on call $24 \mathrm{~h} /$ day. Because the screening of organ donors would be the only absolute indication for NAT testing, a technologist might test a small number of samples monthly and would never expect to see a positive result. This presents issues surrounding the maintenance of competence, a special challenge in the case of NAT testing where laboratory contamination and false-positive results often occur.

The cost of NAT testing for screening is prohibitive. Instrumentation and reagents are expensive, and several technologists need to be specially trained and require ongoing practice to maintain competence. Testing on single samples is usually performed with a number of controls, further increasing the cost. The provision of $24 \mathrm{~h} /$ day callback services represents an additional expense, especially because NAT testing would likely require an additional technologist to return to the

Departments of Pathology, Medicine and Microbiology and Immunology, Dalhousie University, Halifax, Nova Scotia

Correspondence: Dr Kevin Forward, Departments of Pathology, Medicine and Microbiology and Immunology, Dalhousie University,

5788 University Avenue, Room 315, Mackenzie Building, Halifax, Nova Scotia B3H 1V8. Telephone 902-473-4109, fax 902-473-4432,

e-mail kevin.forward@cdha.nshealth.ca

Received and accepted for publication September 29, 2004. 
laboratory for the West Nile NAT test. This situation differs considerably from the Canadian Blood Services situation, in which large numbers of tests are performed at the same time by regularly scheduled technologists. Samples are tested in pools of six patient samples per well; overall costs might be one-fiftieth of that in an organ donor screening program. In addition, there is ample time to retest samples, and the consequences of falsepositive tests are far less disastrous.

Clearly, there is a desire by all to avoid the transmission of WNV to immunocompromised organ recipients. The Organ Procurement and Transplant Network has recently announced that screening for WNV in deceased organ donors is not recommended at this time. To date, the United States Centers for Disease Control and Prevention and the United States Food and Drug Administration have not recommended changes to existing organ donor screening and testing practices. In minimizing the risk associated with a failure to produce a screening directive, Health Canada appears to have minimized the risk to itself. On the other hand, by complying with the recommendations, organ transplant programs will accept a dramatically increased risk associated with testing if false-positive tests lead to organ refusal (as has already been the case). Programs not implementing NAT-based screening accept the liability of not doing so in the face of national guidelines.

In issuing this guidance document, Health Canada has minimized the risk to itself. Physicians must minimize the risk to patients. "First, do no harm!"

\section{REFERENCES}

1. Health Canada. Guidance For Industry: Measures to Prevent West Nile Virus Transmission Through Cells, Tissues and Organs for Transplantation and Assisted Reproduction. <http://www.hcsc.gc.ca/hpfb-dgpsa/bgtd-dpbtg/wnv2004_ctoguidance_e.pdf > (Version current at November 4, 2004).

2. Iwamoto M, Jernigan DB, Gausch A, et al. Transmission of West Nile virus from an organ donor to four transplant recipients. N Engl J Med 2003;348;2196-203.

3. Kiberd BA, Forward K. Screening for West Nile virus in organ transplantation: A medical decision analysis. Am J Transplant 2004;4:1296-301. 


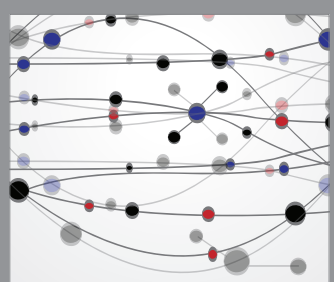

The Scientific World Journal
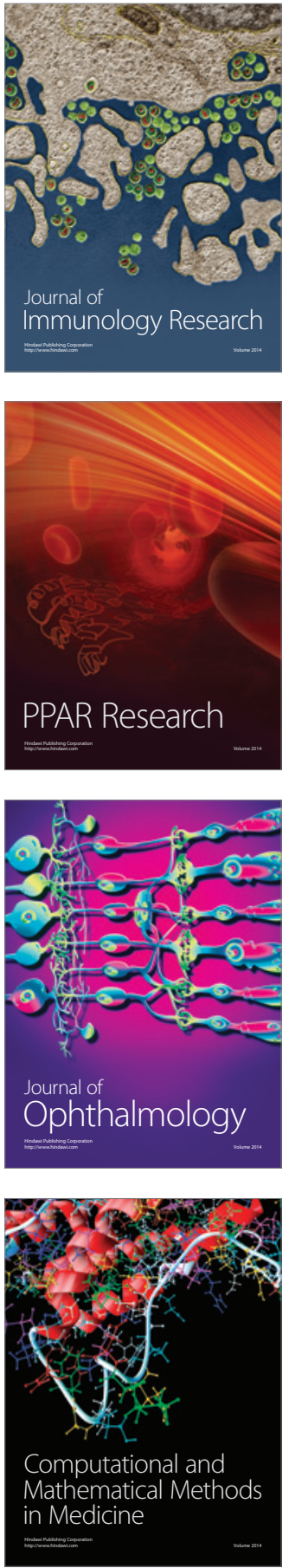

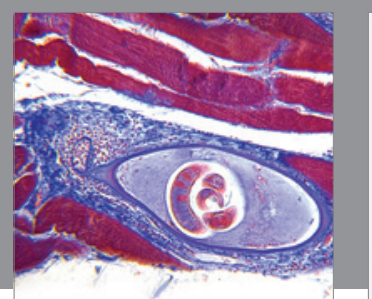

Gastroenterology Research and Practice

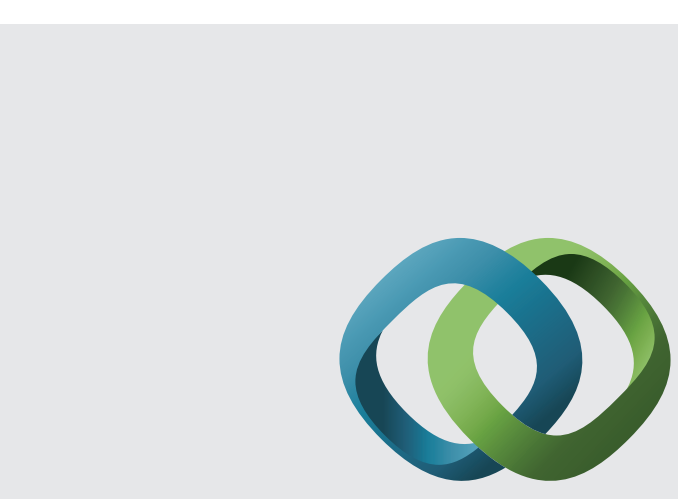

\section{Hindawi}

Submit your manuscripts at

http://www.hindawi.com
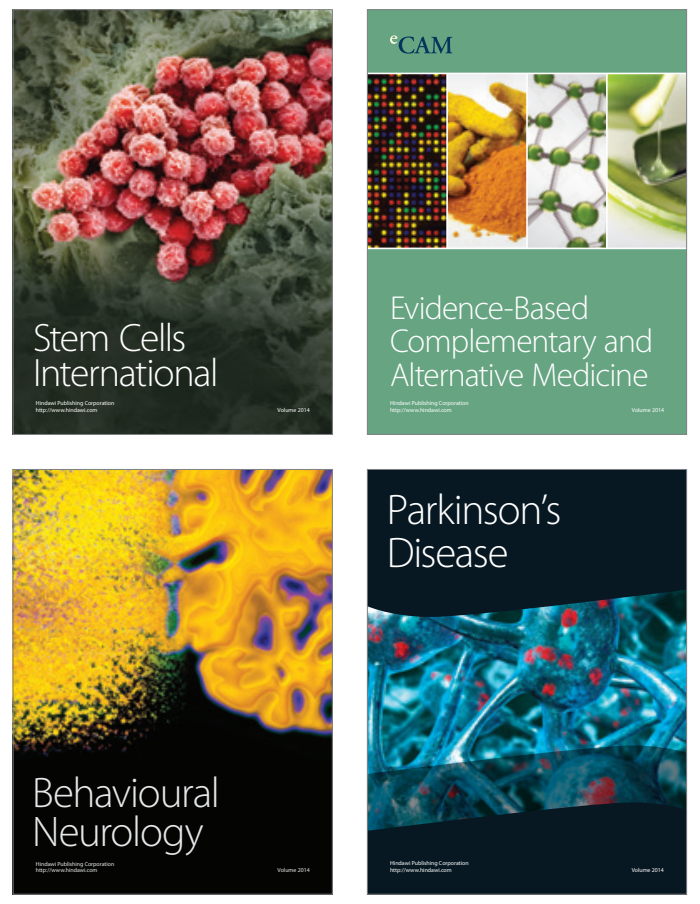
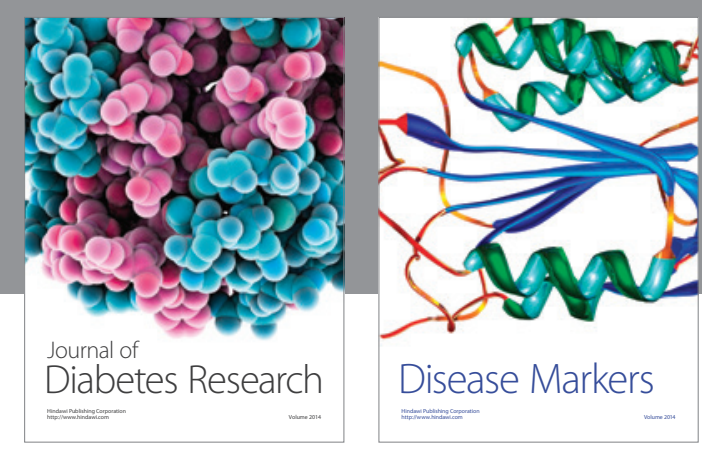

Disease Markers
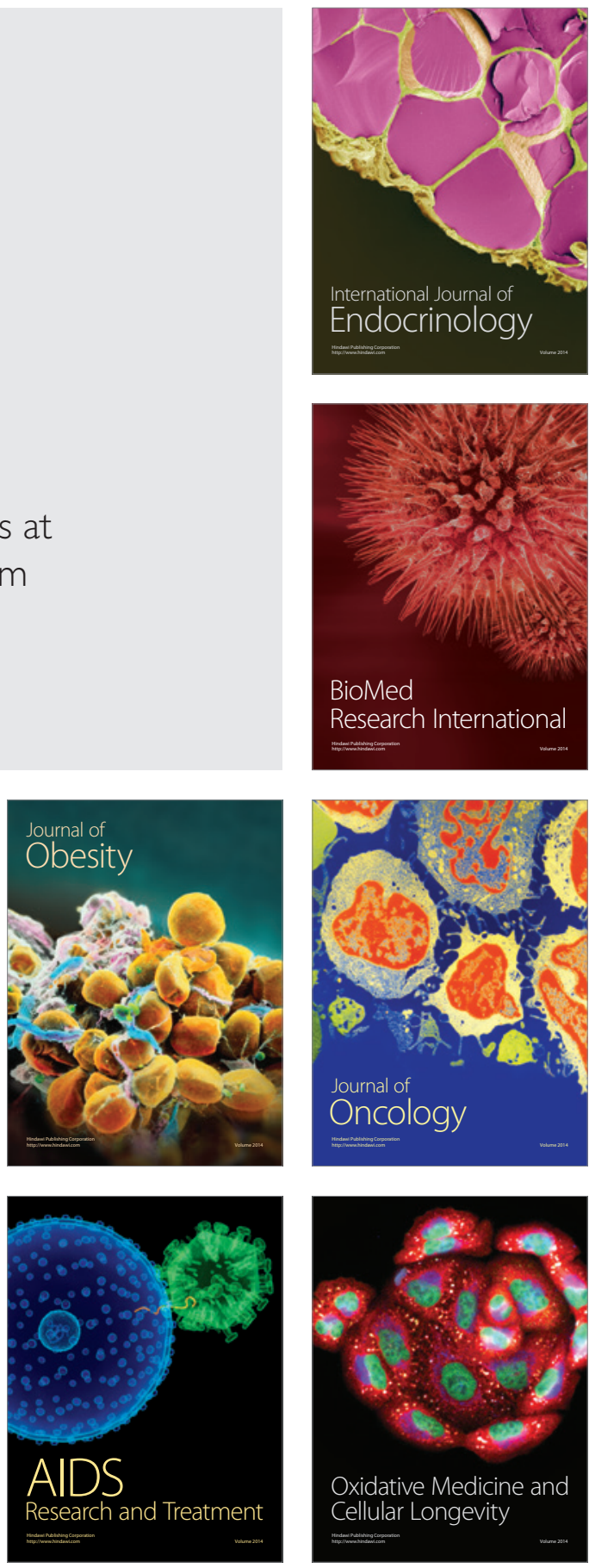